\title{
Bound on dissipative effects from semileptonic neutral $B$-meson decays
}

\author{
F. Benatti ${ }^{1,2}$, R. Floreanini ${ }^{2, a}$, S. Marcantoni ${ }^{1,2}$, P. Pinotti ${ }^{1,2}$, K. Zimmermann ${ }^{1,2}$ \\ ${ }^{1}$ Dipartimento di Fisica, Università di Trieste, 34151 Trieste, Italy \\ ${ }^{2}$ Istituto Nazionale di Fisica Nucleare, Sezione di Trieste, 34151 Trieste, Italy
}

Received: 24 July 2017 / Accepted: 16 September 2017 / Published online: 27 September 2017

(C) The Author(s) 2017. This article is an open access publication

\begin{abstract}
The semileptonic decay asymmetry $\mathcal{A}_{\Delta m}$ is studied within the open quantum systems approach to the physics of the neutral meson $B^{0}-\overline{B^{0}}$ system: this extended treatment takes into account possible non-standard, dissipative effects induced by the presence of an external environment. A bound on these effects is provided through the analysis of available experimental data from the Belle Collaboration.
\end{abstract}

\section{Introduction}

Elementary particle physics is usually formulated using standard quantum mechanics, considering all systems as isolated from the external environment. This general framework cannot, however, accommodate all phenomena involving elementary particles, specifically those leading to decoherence and irreversibility. An extension of the standard treatment is needed in order to properly describe these effects: it can be physically motivated within the so-called open system approach to quantum systems [1-7].

Quite in general, an open quantum system can be modeled as a subsystem $\mathcal{S}$ immersed in an external, large environment $\mathcal{E}$. Although the time evolution of the global system $\mathcal{S}+\mathcal{E}$ follows the rules of ordinary quantum mechanics, that of the subsystem alone, obtained by eliminating the environment degrees of freedom, is no longer unitary and in general rather involved, due to the exchange of energy and entropy between $\mathcal{S}$ and $\mathcal{E}$.

The description greatly simplifies when the interaction between subsystem and environment can be considered to be weak, a rather common situation in actual applications. In this case a mathematically precise modeling of the time evolution of $\mathcal{S}$ alone can be given in terms of linear evolution maps, the so-called quantum dynamical semigroups [1]; they automatically satisfy basic physical requirements,

\footnotetext{
a e-mail: floreanini@ts.infn.it
}

like forward in time composition law (semigroup property), entropy increase (irreversibility), complete positivity (it guarantees the physical consistency of the sub-dynamics in any situation [4]).

This open quantum systems formulation represents a rather universal paradigm for describing irreversibility and decoherence phenomena: it has been successfully applied to model noisy and dissipative effects in quantum optics, atomic and molecular systems, with applications to quantum information and quantum technology $[1,8-10]$.

In the same vein, it can be applied to study irreversibility and decoherence phenomena in elementary particle systems, as these systems can be considered isolated from the environment only in an idealized setting. Indeed, more and more investigations point to the intriguing possibility that quantum gravity effects at Planck's scale or more in general, the microscopic dynamics of extended, fundamental objects (strings and branes) could effectively act as an external environment, inducing non-standard, dissipative effects at low energies. ${ }^{1}$ These new, non-standard phenomena are nevertheless expected to be very small in magnitude and thus difficult to detect: they are suppressed by at least one inverse power of the Planck mass, as a rough dimensional estimate reveals [14]; in spite of this, they can affect interference phenomena thus becoming within the reach of actual detector set-ups.

In this respect, dedicated neutral meson experiments, both at colliders and meson factories, appear to be particularly promising. Indeed, suitable neutral meson observables turn out to be particularly sensible to the new, dissipative effects, so that their presence can be experimentally probed quite independently from other, non-standard phenomena [15-17].

\footnotetext{
1 The original idea that at the Planck scale quantum fluctuations of the space geometry could destroy the smoothness of the space time manifold has been introduced in [11] and, since then, further discussed by many authors; for recent reviews see $[12,13]$ and the references therein.
} 
For completeness, let us mention that the neutral $B$-meson system has been the focus of investigations involving the study of other non-standard effects. In particular, efforts [1820] have been made to provide upper bounds on the so-called "decoherence parameter", originally introduced in [21]; this constant parametrizes a possible modification of the interference term that appears in the expression of quantum mechanical probabilities as a consequence of the superposition principle. As such, this parameter is of kinematical character, not involving directly the $B$-meson dynamics. In addition, investigations adopting a non-standard time evolutions for the neutral $B$-meson system have also appeared in the literature [22,23]; however, those dynamics appear to be postulated in advance, with little physical insight. In this respect, our study, based on the theory of open quantum systems, appears to be rather distinct from those already present in the literature, and the dissipative effects discussed here have essentially no bearings with the ones presented in [18-23].

More specifically, in the present work, we shall discuss in detail how dissipative phenomena can affect the dynamics of the meson $B^{0}-\overline{B^{0}}$ system by focusing on a specific experimentally accessible observable, the time dependent flavor asymmetry of semileptonic $B^{0}$ decays $\mathcal{A}_{\Delta m}$, that has been the focus of intensive experimental studies at $B$-factories in the recent years [24]. In particular, the Belle Collaboration has made available binned, raw data for this asymmetry, corrected for experimental inefficiencies, with both statistical and systematic uncertainties [25]. As we shall see, these data, although limited, will be able to provide interesting bounds on one of the constants parametrizing the dissipative effects. In view of the large improvements in the determination of $\mathcal{A}_{\Delta m}$ and other neutral $B$-meson observables that are expected from $\mathrm{LHCb}$ and Belle II experiments, we are confident that our results will stimulate further interest and additional, more refined studies on possible dissipative effects in neutral meson physics.

\section{Dissipative dynamics for the neutral meson system}

Within the familiar effective approach, the study of the propagation and decay of neutral $B^{0}, \overline{B^{0}}$ mesons requires a twodimensional Hilbert space [26,27]. Meson states are represented by $2 \times 2$ density matrices, i.e. hermitian, positive operators (i.e. with positive eigenvalues), with constant trace (at least for unitary evolutions). In the $\left|B^{0}\right\rangle,\left|\overline{B^{0}}\right\rangle$ basis, any neutral meson state can then be written as

$\rho=\left[\begin{array}{ll}\rho_{1} & \rho_{3} \\ \rho_{4} & \rho_{2}\end{array}\right]$,

where $\rho_{4} \equiv \rho_{3}^{*}$, with $*$ signifying complex conjugation. In the framework of open systems, the time evolution of the state $\rho$ is realized in terms of linear transformations generated by an equation of the form [15-17]:

$\frac{\partial \rho(t)}{\partial t}=-i \mathfrak{H} \rho(t)+i \rho(t) \mathfrak{H}^{\dagger}+\mathfrak{L}[\rho]$

The first two pieces on the r.h.s. give the standard hamiltonian contribution, while $\mathfrak{L}$ is a linear map that takes into account the dissipative, non-standard effects.

The effective hamiltonian $\mathfrak{H}$ includes a non-hermitian part,

$\mathfrak{H}=M-\frac{i}{2} \Gamma$,

with $M$ and $\Gamma$ positive hermitian matrices, which characterizes the masses and natural widths of the $B$-states. The entries of $\mathfrak{H}$ can be expressed in terms of its eigenvalues: $\lambda_{H}=m_{H}-\frac{i}{2} \gamma_{H}, \lambda_{L}=m_{L}-\frac{i}{2} \gamma_{L}$, and the complex parameters $p_{H}, q_{H}, p_{L}, q_{L}$, appearing in the corresponding (right) eigenstates,

$$
\begin{array}{ll}
\left|B_{H}\right\rangle=p_{H}\left|B^{0}\right\rangle+q_{H}\left|\overline{B^{0}}\right\rangle, & \left|p_{H}\right|^{2}+\left|q_{H}\right|^{2}=1, \\
\left|B_{L}\right\rangle=p_{L}\left|B^{0}\right\rangle-q_{L}\left|\overline{B^{0}}\right\rangle & \left|p_{L}\right|^{2}+\left|q_{L}\right|^{2}=1 .
\end{array}
$$

Even in absence of the additional piece $\mathfrak{L}[\rho]$ in (2), probability is not conserved during the time evolution: $\mathrm{d} \operatorname{Tr}[\rho(t)] / \mathrm{d} t \leq 0$. This is due to the presence of a nonhermitian part in the effective hamiltonian $\mathfrak{H}$. On the other hand, loss of phase coherence shows up only when the piece $\mathfrak{L}[\rho]$ is nonvanishing: it produces dissipation and transitions from pure states to statistical mixtures.

Interestingly, quite independently from the details of the dynamics in the environment, the form of the map $\mathfrak{L}[\rho]$ is uniquely fixed by the general physical requirements that the time evolution it generates should satisfy. First of all, the one parameter $\left(=\right.$ time) family of linear maps $\Phi_{t}$, leading from $\rho(0)$ to $\rho(t)=\Phi_{t}[\rho(0)]$, should transform $B$-meson states into $B$-meson states, and therefore should map an initial density matrix $\rho(0)$ into a density matrix $\rho(t)$ at time $t$; further, it should have the property of increasing the (von Neumann) entropy, $S=-\operatorname{Tr}[\rho(t) \ln \rho(t)]$, of obeying the (semigroup) composition law, $\Phi_{t}\left[\rho\left(t^{\prime}\right)\right]=\rho\left(t+t^{\prime}\right)$, for $t, t^{\prime} \geq 0$, of preserving the positivity of $\rho(t)$ for all times. Actually, in the case of correlated $B$-meson systems, this last property requires the time evolution $\Phi_{t}$ to be completely positive, a much restrictive condition than simple positivity [1-4].

These requirements allow the linear map $\mathfrak{L}[\rho]$ to be fully parametrized in terms of six real constants, $a, b, c, \alpha, \beta$ and $\gamma$, of dimension of energy, with $a, \alpha, \gamma$ non-negative; they are not all independent: the above mentioned property of complete positivity requires that they satisfy suitable inequalities 
$[15,16]$. These parameters are determined by the time correlations in the environment; as such, they encode its characteristic physical properties and can be deduced once the microscopic dynamics in the environment is given. Here, instead, we take an effective attitude and treat them as unknown phenomenological parameters, to be determined by the experiment.

A rough evaluation on the magnitude of the dissipative effects induced by $\mathfrak{L}[\rho]$ can be given on the basis of a general dimensional estimate: they should be proportional to powers of the typical energy of the system that we study, while suppressed by inverse powers of the characteristic energy scale of the environment. Following the general idea that dissipation is induced by quantum effects at a large, fundamental scale $M_{F}$ [14], an upper bound on the magnitude of the parameters $a, b, c, \alpha, \beta$ and $\gamma$ can be roughly evaluated to be of order $m^{2} / M_{F}$, where $m$ is the neutral $B$-meson mass. If the non-standard effects have a gravitational origin, the scale $M_{F}$ should coincide with the Planck mass $M_{P}$, and the previous upper bound would give: $m^{2} / M_{P} \sim 10^{-18} \mathrm{GeV} .^{2}$

The behavior in time of physical observables related to the various neutral meson decay channels can be obtained by solving the evolution Eq. (2) for an arbitrary initial state $\rho(0)$. To this aim, it is convenient to use a vector notation and write the matrix $\rho$ as the four-dimensional vector $|\rho\rangle$, with components $\left(\rho_{1}, \rho_{2}, \rho_{3}, \rho_{4}\right)$. Then the evolution Eq. (2) takes the form of a diffusion equation:

$\frac{\mathrm{d}}{\mathrm{d} t}|\rho(t)\rangle=[\mathcal{H}+\mathcal{L}]|\rho(t)\rangle$.

In the basis in which the hamiltonian contribution is diagonal,

$$
\mathcal{H}=-\operatorname{diag}\left(\gamma_{H}, \gamma_{L}, \Gamma_{-}, \Gamma_{+}\right),
$$

$\Gamma_{ \pm}=\Gamma \pm i \Delta m, \quad \Gamma=\frac{\gamma_{H}+\gamma_{L}}{2}, \quad \Delta m=m_{H}-m_{L}$,

the dissipative part $\mathcal{L}$ is a $4 \times 4$ matrix that can be expressed as follows:

$\mathcal{L}=\Gamma\left[\begin{array}{cccc}-D & D & -C & -C^{*} \\ D & -D & C & C^{*} \\ -C^{*} & C^{*} & -A & B(\Delta m / \Gamma) \\ -C & C & B^{*}(\Delta m / \Gamma) & -A\end{array}\right]$

in terms of two real, $A, D$, and two complex, $B, C$, adimensional parameters, simple linear combinations of the constants $a, b, c, \alpha, \beta$ and $\gamma .^{3}$

\footnotetext{
2 Dissipative effects can be investigated also in other neutral meson systems, and in particular in kaon systems $[15,16,28]$; however, on the basis of the above dimensional estimates, the magnitude of such effects are expected to be further suppressed by a few orders of magnitude due to the smaller mass of the involved mesons.

3 The explicit relations expressing $A, B, C, D$ in terms of $a, b, c, \alpha, \beta$ and $\gamma$ can be found in $[17,29]$.
}

Since, as already observed, the contribution of $\mathcal{L}$ is expected to give very small effects, one can solve (5) in perturbation theory,

$|\rho(t)\rangle=\mathrm{e}^{\mathcal{H} t}|\rho(0)\rangle+\int_{0}^{t} \mathrm{~d} s \mathrm{e}^{\mathcal{H}(t-s)} \mathcal{L} \mathrm{e}^{\mathcal{H} s}|\rho(0)\rangle+\ldots$,

retaining only the lowest order terms in the dissipative parameters. In this way, one can find the time dependence of the components $\rho_{1}(t), \rho_{2}(t), \rho_{3}(t), \rho_{4}(t)$ of the vector $|\rho(t)\rangle$, and hence of the density matrix $\rho(t)[17,29,30]$.

These results allow an explicit study of the time evolution of any physical observable of the $B^{0}-\overline{B^{0}}$ system. In the formalism of density matrices, they are described by suitable hermitian operators $\mathcal{O}$. Of particular interest are those observables $\mathcal{O}_{f}$ that are associated with the decay of a neutral meson into final states $f$. In the $\left|B^{0}\right\rangle,\left|\overline{B^{0}}\right\rangle$ basis, $\mathcal{O}_{f}$ is represented by a $2 \times 2$ matrix,

$\mathcal{O}_{f}=\left[\begin{array}{ll}\mathcal{O}_{1} & \mathcal{O}_{3} \\ \mathcal{O}_{4} & \mathcal{O}_{2}\end{array}\right]$

whose entries can be explicitly written in terms of the two independent decay amplitudes $\mathcal{A}\left(B^{0} \rightarrow f\right)$ and $\mathcal{A}\left(\overline{B^{0}} \rightarrow f\right)$ :

$$
\begin{array}{ll}
\mathcal{O}_{1}=\left|\mathcal{A}\left(B^{0} \rightarrow f\right)\right|^{2}, & \mathcal{O}_{3}=\left[\mathcal{A}\left(B^{0} \rightarrow f\right)\right]^{*} \mathcal{A}\left(\overline{B^{0}} \rightarrow f\right), \\
\mathcal{O}_{2}=\left|\mathcal{A}\left(\overline{B^{0}} \rightarrow f\right)\right|^{2}, & \mathcal{O}_{4}=\mathcal{A}\left(B^{0} \rightarrow f\right)\left[\mathcal{A}\left(\overline{B^{0}} \rightarrow f\right)\right]^{*} .
\end{array}
$$

Its mean value is a physical quantity, directly accessible to the experiment, whose time evolution is given by

$\left\langle\mathcal{O}_{f}\right\rangle(t) \equiv \operatorname{Tr}\left[\mathcal{O}_{f} \rho(t)\right]$

This general formula can be used to explicitly compute experimentally relevant decay rates and asymmetries.

Although, as already mentioned, the basic general idea behind the open system approach to $B$-meson dynamics is that quantum phenomena at Planck's scale could produce loss of phase coherence, it should be stressed that the form (5)-(8) of the evolution equation is quite independent from the microscopic mechanism responsible for the dissipative effects; indeed, as already remarked, quite in general the evolution of any quantum irreversible process can be effectively modeled in terms quantum dynamical semigroups. In this respect, the dynamics generated by (5) is the best suited for experimental tests: any signal of a non-vanishing value for some of the parameters appearing in (8) would attest in a model independent way the presence of non-standard, dissipative effects in $B$-meson physics. ${ }^{4}$

\footnotetext{
4 In this respect, we stress again that our attitude is quite different from that of other recent investigations on "decoherence" effects in $B$-meson physics, e.g. see $[22,23,31]$, where ad hoc dynamics are postulated in advance.
} 


\section{Correlated neutral $B$-mesons semileptonic decays}

The non-standard, dissipative effects described by the generalized dynamics (5) can be most effectively analyzed in experiments involving correlated neutral mesons, at meson factories; indeed, being quantum mechanical interferometers, these set-ups appear particularly suitable for detecting tiny phenomena related to loss of quantum coherence.

In those experiments, correlated $B^{0}-\overline{B^{0}}$ mesons are produced from the decay of the $\Upsilon(4 S)$ spin-1 meson resonance [24]. Since it decays into two spinless bosons, it produces an antisymmetric spatial state. In the resonance-rest frame, the two neutral mesons are produced flying apart with opposite momenta $\vec{p}$; in the $\left|B^{0}\right\rangle,\left|\overline{B^{0}}\right\rangle$ basis, the resulting state can then be described by

$\left|\psi_{A}\right\rangle=\frac{1}{2}\left(\left|B^{0},-\vec{p}\right\rangle \otimes\left|\overline{B^{0}}, \vec{p}\right\rangle-\left|\overline{B^{0}},-\vec{p}\right\rangle \otimes\left|B^{0}, \vec{p}\right\rangle\right)$.

The corresponding density operator $\rho_{A}$ is a $4 \times 4$ matrix and it is given by the projector onto the above two-meson state: $\rho_{A}=\left|\psi_{A}\right\rangle\left\langle\psi_{A}\right|$. Its evolution in time can be analyzed using the previously discussed single-meson dynamics: once produced in a resonance-decay the two mesons can be considered independent and evolve in time each according to the completely positive map generated by (5). This guarantees that the resulting evolution is completely positive and of semigroup type, therefore ensuring a physically meaningful dynamics at all times.

As explicitly shown by (12), the two neutral $B$-mesons that come from a decay of the $\Upsilon(4 S)$ resonance are quantummechanically entangled, in a way very similar to that of two spin $1 / 2$ particles coming from a singlet state [9]. As in that case, correlated measures on the two particles become physically significant. Indeed, the typical observables that can be studied at a generic meson factory are double decay rates, i.e. the probabilities $\mathcal{P}\left(f_{1}, t_{1} ; f_{2}, t_{2}\right)$ that a meson decays into a final state $f_{1}$ at proper time $t_{1}$, while the other meson decays into the final state $f_{2}$ at proper time $t_{2}$. They can be computed using

$\mathcal{P}\left(f_{1}, t_{1} ; f_{2}, t_{2}\right)=\operatorname{Tr}\left[\left(\mathcal{O}_{f_{1}} \otimes \mathcal{O}_{f_{2}}\right) \rho_{A}\left(t_{1}, t_{2}\right)\right]$,

where $\mathcal{O}_{f_{1}}, \mathcal{O}_{f_{2}}$ represent $2 \times 2$ hermitian matrices describing the decay of a single meson into the final states $f_{1}, f_{2}$, respectively, while $\rho_{A}\left(t_{1}, t_{2}\right)$ is the time evolution of the initial density matrix $\rho_{A}=\left|\psi_{A}\right\rangle\left\langle\psi_{A}\right|$ up to $t_{1}$ for the first meson and up to $t_{2}$ for the second.

In practice, in the case of the neutral $B$-mesons, the short lifetime and rapid $B^{0}-\overline{B^{0}}$ oscillations do not allow a precise enough study of the double time dependence in (13). Indeed, much of the analysis at $B$-meson factories is carried out using integrated distributions at fixed time interval $t=t_{1}-t_{2}$ [24].
One then focuses on single-time distributions, defined by

$\Gamma\left(f_{1}, f_{2} ; t\right) \equiv \int_{0}^{\infty} \mathrm{d} t^{\prime} \mathcal{P}\left(f_{1}, t^{\prime}+t ; f_{2}, t^{\prime}\right), \quad t \geq 0$,

while, for negative $t$, one has $\Gamma\left(f_{1}, f_{2} ;-|t|\right)=\Gamma\left(f_{2}, f_{1} ;|t|\right)$.

It should be stressed that, in the presence of an external environment, the behavior of $\Gamma\left(f_{1}, f_{2} ; t\right)$ is in general quite different from that obtained in the standard case. The most striking difference arises when the final states coincide $f_{1}=$ $f_{2}=f$ and $t$ approaches zero. Due to the antisymmetric character of the initial state $\left|\psi_{A}\right\rangle$ in (12), standard unitary evolution predicts a vanishing value for $\Gamma(f, f ; 0)$, while in general this is not the case for the completely positive dynamics generated by (5). This result reinforces the use of correlated mesons systems for studying effects leading to loss of phase coherence and dissipation.

We shall now consider observables connected with $B$ meson decays into semileptonic final states, $h \ell v$, where $h$ stands for any allowed charged hadronic state, while $\ell$ is a charged lepton and $v$ its corresponding neutrino. More specifically, we shall focus on the asymmetry

$\mathcal{A}_{\Delta m}(t)$

$$
=\frac{\left[\Gamma\left(h^{+}, h^{-} ; t\right)+\Gamma\left(h^{-}, h^{+} ; t\right)\right]-\left[\Gamma\left(h^{+}, h^{+} ; t\right)+\Gamma\left(h^{-}, h^{-} ; t\right)\right]}{\Gamma\left(h^{+}, h^{+} ; t\right)+\Gamma\left(h^{-}, h^{-} ; t\right)+\Gamma\left(h^{+}, h^{-} ; t\right)+\Gamma\left(h^{-}, h^{+} ; t\right)},
$$

which turns out to be particularly sensible to the nonstandard, dissipative effects. As mentioned before, this asymmetry has been the target of intense experimental studies, as it is one of the preferred observables for obtaining a precise determination of the $B$-meson mass difference $\Delta m$ [32].

Let us point out that the evolution equation (5) is manifestly invariant under a phase change of the basis states $\left|B^{0}\right\rangle,\left|\overline{B^{0}}\right\rangle$; further, no commitment on possible violations of discrete symmetries $(C P, T$ and $C P T)$ or of the socalled $\Delta B=\Delta Q$ rule have so far been made. Actually, the dependence of the asymmetry $\mathcal{A}_{\Delta m}$ (and in fact of the other $B$-meson observables) on the dissipative parameters is very distinctive and quite different from that of the parameters violating the above mentioned discrete symmetries. In other terms, the appearance of environment-induced dissipative phenomena can be studied independently from the other, more familiar symmetry-violating effects.

Nevertheless, for the analysis of the data presented in [25], we shall adopt the same attitude usually taken in the experimental determination of the mass difference $\Delta m$, assuming no $C P T$ - and $C P$-violations in mixing and the validity of the $\Delta B=\Delta Q$ rule. In this case, the semileptonic $B$-meson decays become "flavor tagging", since the decay channels $B^{0} \rightarrow h^{+} \ell^{-} \bar{v}$ and $\overline{B^{0}} \rightarrow h^{-} \ell^{+} v$ are forbidden. In other terms, by looking at the charge of the decay products, one can reconstruct the flavor of the original decayed meson. 
Table 1 Time-binned data set for the asymmetry $\mathcal{A}_{\Delta m}$ as given in [25]

\begin{tabular}{lcc}
\hline Bin & $t_{\text {in }}-t_{\text {fin }}(\mathrm{ps})$ & $\mathcal{A}_{\Delta m}$ and total error \\
\hline 1 & $0.0-0.5$ & $1.013 \pm 0.028$ \\
2 & $0.5-1.0$ & $0.916 \pm 0.022$ \\
3 & $1.0-2.0$ & $0.699 \pm 0.038$ \\
4 & $2.0-3.0$ & $0.339 \pm 0.056$ \\
5 & $3.0-4.0$ & $-0.136 \pm 0.075$ \\
6 & $4.0-5.0$ & $-0.634 \pm 0.084$ \\
7 & $5.0-6.0$ & $-0.961 \pm 0.077$ \\
8 & $6.0-7.0$ & $-0.974 \pm 0.080$ \\
9 & $7.0-9.0$ & $-0.675 \pm 0.109$ \\
10 & $9.0-13.0$ & $0.089 \pm 0.193$ \\
11 & $13.0-20.0$ & $0.243 \pm 0.435$ \\
\hline
\end{tabular}

One can than group the single-time integrated distributions appearing in (15) into "same flavor", $\Gamma^{(S F)}(t)$ $=\Gamma\left(h^{+}, h^{+} ; t\right)+\Gamma\left(h^{-}, h^{-} ; t\right)$, and "opposite flavor", $\Gamma^{(O F)}(t)=\Gamma\left(h^{+}, h^{-} ; t\right)+\Gamma\left(h^{-}, h^{+} ; t\right)$, final state decay rates, so that

$\mathcal{A}_{\Delta m}(t)=\frac{\Gamma^{(O F)}(t)-\Gamma^{(S F)}(t)}{\Gamma^{(O F)}(t)+\Gamma^{(S F)}(t)}$.

The combinations $\Gamma^{(i)}(t), i=S F, O F$, are the quantities directly measured by the experiment, combined in the asymmetry (16) in order to reduce systematic uncertainties. Note that, due to the decay of the neutral $B$ mesons, the useful events become necessarily rarer and rarer as the time difference $t$ between the two-meson decays increases. To cope with this drawback, one usually group the data in suitable timebins, with increasing width. The original data set provided in [25] is reproduced in Table 1: for each bin $n$, the two bin extrema, $t_{\text {in }}^{(n)}-t_{\text {fin }}^{(n)}$, the measured asymmetry and the associated combined statistical plus systematic error are explicitly reported.

These data can now be used to fit the expression of the asymmetry obtained from the solution of the dissipative timeevolution equation (5). As the data in Table 1 are limited, we shall confine the discussion to a model in which the dissipative parameter $a$ is vanishing: complete positivity then imposes $\alpha=\gamma$ and $c=b=\beta=0$, so that the dissipative contribution $\mathcal{L}$ in (8) can be expressed in terms of the single adimensional constant $A=\alpha / \Gamma$, since $B=D=A$ and $C=0$ [17]. This choice further ensures invariance under both $C P T$ and $T$ transformations also in the dissipative part $\mathcal{L}$ of the evolution equation (5).

Explicit computation then gives [17,29]

$$
\begin{aligned}
& \Gamma^{(O F)}(t)-\Gamma^{(S F)}(t) \\
= & \mathrm{e}^{-\Gamma t}\left\{\left(1+\frac{A}{1+\frac{\Delta m^{2}}{\Gamma^{2}}}\right) \cos (\Delta m t)+\frac{A}{\frac{\Delta m}{\Gamma}\left(1+\frac{\Delta m^{2}}{\Gamma^{2}}\right)} \sin (\Delta m t)\right\},
\end{aligned}
$$

$\Gamma^{(O F)}(t)+\Gamma^{(S F)}(t)$

$=\mathrm{e}^{-\Gamma t}\left\{(1+A) \cosh \left(\frac{\Delta \Gamma}{2} t\right)+\frac{2 \Gamma A}{\Delta \Gamma} \sinh \left(\frac{\Delta \Gamma}{2} t\right)\right\}$,

where, for generality, we have kept non-vanishing the decay width difference $\Delta \Gamma=\gamma_{H}-\gamma_{L}$. Neglecting dissipative effects, $A=0$, and assuming $\Delta \Gamma=0$, from the above formulas one readily obtain the usual expression for the asymmetry, $\mathcal{A}_{\Delta m}(t)=\cos (\Delta m t)$, used at meson factories for the precise determination of the mass difference $\Delta m$.

The expressions in (17) cannot be directly used for fitting purposes, as the data in Table 1 do not give the magnitude of the asymmetry as the ratio of instantaneous decay rates, rather as the ratio of the decay probabilities within the given interval of time defining each bin; these probabilities are proportional to the number of the relevant decay events that fall in the corresponding bins. Then, in order to compare the experimental data given Table 1 with the theoretical predictions, one should first integrate the decay rates $\Gamma^{(i)}(t)$, $i=S F, O F$, along the time interval defining each of the 11 data-bins,

$\Gamma^{(i)}\left(t_{\text {in }}^{(n)}, t_{\mathrm{fin}}^{(n)}\right)=\int_{t_{\mathrm{in}}^{(n)}}^{t_{\mathrm{fin}}^{(n)}} \mathrm{d} t \Gamma^{(i)}(t), \quad n=1,2, \ldots, 11$

and from them reconstruct the corresponding values for the asymmetry:

$\mathcal{A}_{\Delta m}^{(n)}=\frac{\Gamma^{(O F)}\left(t_{\mathrm{in}}^{(n)}, t_{\mathrm{fin}}^{(n)}\right)-\Gamma^{(S F)}\left(t_{\mathrm{in}}^{(n)}, t_{\mathrm{fin}}^{(n)}\right)}{\Gamma^{(O F)}\left(t_{\mathrm{in}}^{(n)}, t_{\mathrm{fin}}^{(n)}\right)+\Gamma^{(S F)}\left(t_{\mathrm{in}}^{(n)}, t_{\mathrm{fin}}^{(n)}\right)}$

these are the quantities to be compared with the experimental data in the third column of Table 1.

As a consistency check of the whole procedure, we first analyzed the data set of Table 1 under the hypothesis of vanishing dissipative effects, $A=0$, assuming $\Delta \Gamma=$ $10^{-2} \mathrm{ps}^{-1}$, a value consistent both with the predictions of the Standard Model and the present experimental estimations, leaving only the mass difference $\Delta m$ as an unknown. Performing a least square fit using the Root package, we obtained $\Delta m=0.504 \pm 0.009 \mathrm{ps}^{-1}$, with $\chi^{2}=5.0$ for ten degrees of freedom, a value perfectly compatible with the one given by the Belle Collaboration [25] and the world average [32].

We then performed a two-parameter fit of the data in Table 1, keeping both the dissipative constant $A$ and the mass difference $\Delta m$ as free parameters, while fixing the width $\Gamma$ to its world average value [32], and assuming again $\Delta \Gamma=10^{-2} \mathrm{ps}^{-1}$. The result of the fit gives for $\Delta m$ the same value as before, while for the dissipative parameter one gets

$A=(1.5 \pm 8.4) \cdot 10^{-3}$ 


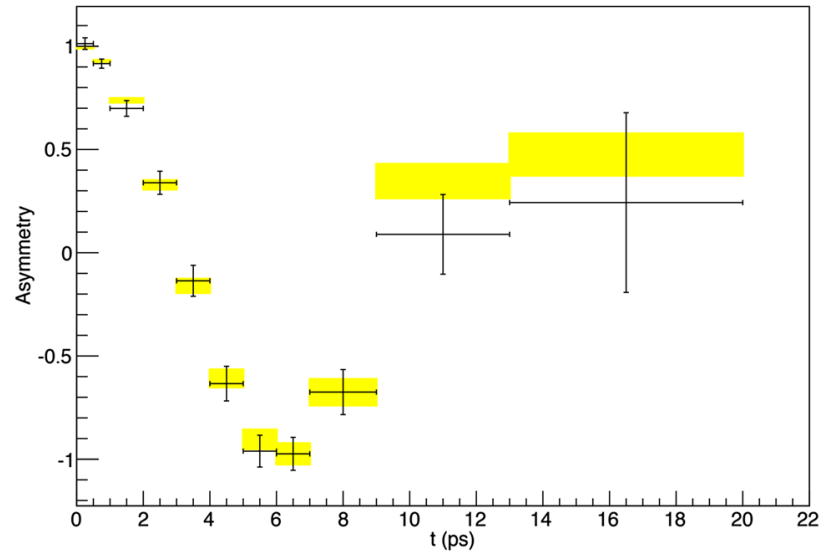

Fig. 1 Asymmetry $\mathcal{A}_{\Delta m}$ : experimental data (crosses) and the result of the least-squares fit (yellow boxes, showing $\pm 1 \sigma$ combined errors on the dissipative parameter $A$ and $\Delta m$ )

with $\chi^{2}=4.9$ for nine degrees of freedom; the correlation between the two fitted parameters is also low, and it is given by $C(\Delta m, A)=0.14$. In Fig. 1 the outcome of the fit is compared with the experimental data, showing also visually their agreement.

Although compatible with zero, the obtained value for the parameter $A$ represents one of the best tests so far available of the presence of dissipative effects in elementary particle physics. Indeed, going back to the original parameter $\alpha=\Gamma A$ entering the dissipative contribution to the evolution equation (2), one can re-express the result (20) as the following upper bound:

$\alpha \leq 7.9 \cdot 10^{-15} \mathrm{GeV} \quad(95 \% \mathrm{CL})$,

not very far from the estimate based on quantum gravity effects mentioned before. The future availability of more accurate data sets and the combined analysis of additional $B$-meson observables will surely improve this result.

\section{Outlook}

The description of open quantum systems in terms of quantum dynamical semigroups provides a very general and physically consistent approach to the study of phenomena leading to irreversibility and dissipation. When applied to the analysis of the propagation and decay of correlated neutral $B$-mesons, it gives precise predictions on the behavior of relevant physical observables: the dissipative phenomena can be parametrized through a set of phenomenological constants, quite independently from the details of the fundamental, microscopic dynamics from which they originate.
Various observables involving the correlated $B^{0}-\overline{B^{0}}$ system can be identified as being particularly sensitive to these new phenomena. In the present investigation, we have focused on the semileptonic decay asymmetry $\mathcal{A}_{\Delta m}$ and used data from the Belle Collaboration to give constraints on the dissipative effects; the obtained upper bound is not too far from estimates based on the hypothesis of a quantum gravity origin of these non-standard phenomena.

This is a first step towards a more thorough investigation of environment-induced effects in meson systems. Indeed, experimental data related to different observables and asymmetries could be readily analyzed with our fully developed theoretical framework. These observables will be measured with great accuracy at dedicated $B$-meson experiments, both at colliders (LHCb) and at $B$-factories (Belle II), so that a much more accurate analysis of the possible presence of dissipative effects in elementary particle physics can surely be expected in the near future. In particular, the richer statistics will surely allow for the thorough study of all six dissipative parameters and the physical implications they underlie.

Open Access This article is distributed under the terms of the Creative Commons Attribution 4.0 International License (http://creativecomm ons.org/licenses/by/4.0/), which permits unrestricted use, distribution, and reproduction in any medium, provided you give appropriate credit to the original author(s) and the source, provide a link to the Creative Commons license, and indicate if changes were made.

Funded by SCOAP ${ }^{3}$.

\section{References}

1. R. Alicki, K. Lendi, in Quantum Dynamical Semigroups and Applications, 2nd edn. Lecture Notes in Physics, vol 717 (Springer, Berlin, 2007)

2. R. Alicki, M. Fannes, Quantum Dynamical Systems (Oxford University Press, Oxford, 2001)

3. R. Alicki, , in Invitation to Quantum Dynamical Semigroups. Lecture Notes in Physics, vol 597 (Springer, Berlin, 2002), p. 239

4. F. Benatti, R. Floreanini, Open quantum dynamics: complete positivity and entanglement. Int. J. Mod. Phys. B 19, 3063 (2005)

5. F. Benatti, Dynamics, Information and Complexity in Quantum Systems (Springer, Berlin, 2009)

6. A. Rivas, S. Huelga, Open Quantum Systems (Springer, Berlin, 2012)

7. D. Chruściński, On time-local generators of quantum evolution. Open Syst. Inf. Dyn. 21, 1440004 (2014)

8. C.W. Gardiner, P. Zoller, Quantum Noise (Springer, Berlin, 2004)

9. M.A. Nielsen, I.L. Chuang, Quantum Computation and Quantum Information (Cambridge University Press, Cambridge, 2010)

10. F. Benatti, M. Fannes, R. Floreanini, D. Petritis (eds.), in Quantum Information, Computation and Cryptography. Lecture Notes in Physics, vol 808 (Springer, Berlin, 2010)

11. J.A. Wheeler, Geometrodynamics (Academic Press, New York, 1962)

12. G. Amelino-Camelia, Living Rev. Rel. 16, 5 (2013)

13. A.D.K. Plato, C.N. Hughes, M.S. Kim, Contemp. Phys. 54, 477 (2016)

14. F. Benatti, R. Floreanini, Ann. Phys. 273, 58 (1999) 
15. F. Benatti, R. Floreanini, Nucl. Phys. B 488, 335 (1997)

16. F. Benatti, R. Floreanini, Nucl. Phys. B 511, 550 (1998)

17. F. Benatti, R. Floreanini, R. Romano, Nucl. Phys. B 602, 541 (1998)

18. R.A. Bertlmann, W. Grimus, Phys. Lett. B 392, 426 (1997)

19. G.V. Dass, K.V.L. Sarma, Eur. Phys. J. C 5, 283 (1998)

20. A. Lenz et al., Nucl. Phys. B 888, 129 (2014)

21. P.H. Eberhard, in Tests of Quantum Mechanics at a $\Phi$-Factory. L. Maiani, G. Pancheri, N. Paver (eds.), The Second Daథne Physics Handbook (Frascati, 1995)

22. R.A. Bertlmann, W. Grimus, Phys. Rev. D 64, 056004 (2001)

23. A.K. Alok, S. Banerjee, S.U. Sankar, Phys. Lett. B 749, 94 (2015)

24. A.J. Bevan, B. Golob, Th. Mannel, S. Prell, B.D. Yabsley (eds.), The physics of the $B$ factories. Eur. Phys. J. C 74, 3026 (2014)
25. The Belle Collaboration, Phys. Rev. Lett. 99, 131802 (2007)

26. G.C. Branco, L. Lavoura, J.P. Silva, CP Violation (Clarendon Press, Oxford, 1999)

27. I.I. Bigi, A.I. Silva, CP Violation (Cambridge Univeristy Press, Cambridge, 2000)

28. A. Di Domenico, Found. Phys. 40, 852 (2010)

29. S. Marcantoni, Dissipative effects in neutral B meson systems. Master Thesis, University of Trieste (2014)

30. P. Pinotti, Dissipative B mesons. Bachelor Thesis, University of Trieste(2016)

31. N. Mavromatos, Found. Phys. 40, 917 (2010)

32. C. Patrignani et al., (Particle Data Group), Chin. Phys. C 40, 100001 (2016) 